\title{
Coding of Time-Varying Signals in Spike Trains of Integrate-and-Fire Neurons with Random Threshold
}

\author{
Fabrizio Gabbiani \\ Christof Koch \\ Division of Biology, 139-74 Calted, Pasatena, CA 91125 USA
}

Recently, methods of statistical estimation theory have been applied by Bialek and collaborators (1991) to reconstruct time-varying velocity signals and to investigate the processing of visual information by a directionally selective motion detector in the fly's visual system, the $\mathrm{H1}$ cell. We summarize here our theoretical results obtained by studying these reconstructions starting from a simple model of $\mathrm{H} 1$ based on experimental data. Under additional technical assumptions, we derive a closed expression for the Fourier transform of the optimal reconstruction filter in terms of the statistics of the stimulus and the characteristics of the model neuron, such as its firing rate. It is shown that linear reconstruction filters will change in a nontrivial way if the statistics of the signal or the mean firing rate of the cell changes. Analytical expressions are then derived for the mean square error in the reconstructions and the lower bound on the rate of information transmission that was estimated experimentally by Bialek et al. (1991). For plausible values of the parameters, the model is in qualitative agreement with experimental data. We show that the rate of information transmission and mean square error represent different measures of the reconstructions: in particular, satisfactory reconstructions in terms of the mean square error can be achieved only using stimuli that are matched to the properties of the recorded cell. Finally, it is shown that at least for the class of models presented here, reconstruction methods can be understood as a generalization of the more familiar reverse-correlation technique.

\section{Introduction}

In many animals auditory and visual stimuli are detected by the peripheral sensory system and processed centrally with an appreciable temporal resolution, ranging from typical reaction times of $30 \mathrm{msec}$ in free-flying flies down to the discrimination of time delays in the microsecond range for sound location in barn owls and for echolocating bats (Land and Collett 1974; Simmons 1979; Moiseff and Konishi 1981). The coding of such information in neuronal spike trains and its subsequent elaboration are

Neural Computatim 8, 44-66 (1996) C) 1995 Massachusetts Institute of Technology 
subjects that are still poorly understood. While single neurons might transmit information primarily through their mean firing rate (Adrian 1928), it has also been emphasized that additional information could be encoded in the temporal structure of neuronal spike trains (see for example, Poggio and Viernstein 1964; Miller and Sachs 1983; Optican and Richmond 1987; Bialek et al. 1991; Softky and Koch 1993; Middlebrooks etal. 1994). Collective phenomena in large groups of neurons such as synchronization or temporal oscillations in the correlation of multiple-unit firing rates might as well contribute to the processing of information in central nervous pathways (Eckhorn et al. 1988; Gray et al. 1989). But the relation between single cell codes and neuronal population codes remains unclear.

In this context, it is of interest to study how much information the spike train of a single cell is able to carry about a time-varying stimulus. One approach to investigate this problem consists in presenting repeatedly to the animal a stimulus drawn from an ensemble with known probability distribution while recording the evoked spike trains from a single neuron. By applying methods of stochastic estimation theory (Wiener 1949; Saleh 1978; Poor 1994), it is then possible to compute a temporal filter $h(t)$ that, when convolved with the spike train of the neuron in response to a stimulus $s(t)$, will produce an estimate $s_{\text {est }}(t)$ of $s(t)$. In other words, it is possible to reconstruct part of the time-course of the stimulus from the spike train. Such methods have been introduced into neurobiology by Bialek and collaborators to study the transmission of information by peripheral sensory neurons in a variety of preparations (Bialek et al. 1991; Rieke et al. 1993). These authors have computed a lower bound on the rate of information transmitted by $s_{\text {est }}(t)$ about $s(t)$. In the case of the visual system of the fly, a wide-field horizontal cell of the lobula plate (in the third-order optic ganglia of the fly), the $\mathrm{H} 1$ cell, is capable of transmitting at least $32 \mathrm{bit} / \mathrm{sec}$ of information about a time-varying velocity stimulus presented to the fly.

The reconstructions reported by Bialek et al. (1991) and Rieke et al. (1993) raise several interesting questions. One would first like to know the biological significance of the filter $h$ : it has been suggested that similar algorithms might be implemented at the level of single neurons to decode presynaptic spike trains (Bialek et al. 1991). A related problem is to clarify the connection to neuronal encoding mechanisms as well as to the underlying biophysics of single cells. For example, it is not clear how the properties of the recorded neuron, such as its firing rate, affect estimates of the filter $h(t)$. One would also like to know the relationship between the rate of information transmission of the neurons, as estimated in previous studies, and the measure quantifying the quality of reconstructions, the mean square error. Several issues related to these questions have been addressed in earlier theoretical work (Bialek 1989; Bialek and Zee 1990; Bialek 1992), but the models considered did not allow an explicit calculation of the reconstruction filter $h$. We report here 
that by using different models, it is possible to obtain a closed formula for the reconstruction filter and to compare directly theoretical results with earlier experimental work. More technical details and further theoretical results are contained in Gabbiani (1995).

The rest of this paper is organized as follows: in Section 2, we describe the linear reconstruction of signals from spike trains and derive formulas for the rate of information transmission and the mean square error in the reconstructions. Section 3 introduces the class of models that we are studying, while in Section 4 we present results obtained with a simplified version of these models. Finally, we discuss our results in Section 5.

\section{Linear Estimation of Time-Varying Signals from Neuronal Spike}

\section{Trains}

Let $s_{0}(t)$ be a gaussian random stimulus with finite variance that is presented to the animal. In the experiments performed by Bialek et al. (1991), $s_{0}(t)$ was the velocity of a random pattern presented to the fly: it consisted in a zero mean gaussian white noise signal with cut-off frequency $f_{c}=$ $1000 \mathrm{~Hz}$ and a standard deviation of $132 \mathrm{deg} / \mathrm{sec}$. Let $x_{0}(t)=\sum_{i} \partial\left(t-t_{i}\right)$ be the spike train recorded from the cell. We assume that $s_{0}(t)$ is bandwidth limited with angular cut-off frequency $\alpha_{i}=2 \pi f_{c}$ and that $s_{0}(t)$ and $x_{1}(t)$ are jointly (weakly) stationary.

Let $s_{0}$ and $x_{0}$ be the mean values of $s_{0}(t)$ and $x_{0}(t)$, respectively. In the following we will consider the stimulus and spike train with their mean value subtracted, ${ }^{\prime} s(t)=s_{0}(t)-s_{0}$ and $x(t)=x_{0}(t)-x_{0}$. We write down a linear estimate $s_{\text {est }}(t)$ of the stimulus $s(t)$ given the spike train by setting

$$
s_{\text {est }}(t)=\int d t_{1} h_{l}\left(t_{1}\right) x\left(t-t_{1}\right)
$$

The linear filter $h$ is to be chosen in such a way as to minimize the mean square error between the stimulus and the estimate of the stimulus,

$$
\epsilon(h\}^{2}=\left\langle\left|s(t)-s_{\mathrm{t} s t}(t)\right|^{2}\right\rangle
$$

where the brackets $\langle\cdot\rangle$ mean average over the presented stimuli and recorded spike trains.

If we define the cross-correlation between the stimulus and spike train,

$$
R_{s x}(\tau)=\langle s(t) x(t+\tau)\rangle
$$

the autocorrelation function of the spike train,

$$
R_{m}(\tau)=\langle x(t) x(t+\tau)\rangle
$$

\footnotetext{
'If the stimulus or the spike train sample functions contain other deterministic components, these need to be subtracted as well (see Wiener 1949, sect. 2.4).
} 
and their Fourier transform ${ }^{2}$ through

$$
\begin{aligned}
& S_{s x}(\omega)=\int d \tau R_{s x}(\tau) e^{i \omega \tau}, \\
& S_{x x}(\omega)=\int d \tau R_{x x}(\tau) e^{i \omega \tau},
\end{aligned}
$$

it follows from the orthogonality principle (Poor 1994, sect. V.D.1) that the Fourier transform of the optimal linear filter $h(t)$,

$$
\hat{h}(\omega)=\int d t h(t) e^{i \omega t}
$$

is given by ${ }^{3}$

$$
\widehat{h}(\omega)=\frac{S_{s x}(-\omega)}{S_{x x}(\omega)}
$$

In practice, the Fourier transform $\hat{h}(\omega)$ is approximated numerically by the discrete Fourier transform and $S_{s x}(\omega), S_{x x}(\omega)$ are computed by replacing averages over the stimulus ensemble by time-averaging over a single sample of the ensemble (Oppenheim and Schaffer 1975; Rabiner and Gold 1975).

Since the filter $h$ is derived by minimizing the mean square error between the stimulus and estimated stimulus (see equation 2.1), the natural measure for the quality of reconstructions is the mean square error, $\epsilon^{2}$. Experimentally, a numerically accurate estimate of $\epsilon^{2}$ is obtained from

$$
\begin{aligned}
\left\langle\left|s(t)-s_{\text {est }}(t)\right|^{2}\right\rangle & =\lim _{T \rightarrow \infty} \frac{1}{T} \int_{0}^{T} d t\left[s(t)-s_{\text {est }}(t)\right]^{2} \\
& \cong \frac{1}{N} \sum_{k=1}^{N}\left(s_{k}-s_{\text {est } k}\right)^{2}
\end{aligned}
$$

where $s_{k}$ and $s_{\text {est } k}$ are the sample points of the stimulus and estimated stimulus, respectively. To gain theoretical insight in the dependence of $\epsilon^{2}$ on the choice of the stimulus ensemble, it is convenient to define the "noise" contaminating the reconstructions as the difference between $s_{\text {est }}(t)$ and $s(t)$,

$$
n(t)=s_{\mathrm{est}}(t)-s(t) \Leftrightarrow s_{\mathrm{est}}(t)=s(t)+n(t) .
$$

The mean square error in the reconstructions is then given by

$$
\begin{aligned}
\epsilon^{2} & =\left\langle\left|s(t)-s_{\text {est }}(t)\right|^{2}\right\rangle \\
& =\left\langle|n(t)|^{2}\right\rangle \\
& =\frac{1}{2 \pi} \int_{-\omega_{c}}^{\omega_{c}} d \omega S_{n n}(\omega),
\end{aligned}
$$

\footnotetext{
${ }^{2} S_{x x}(\omega)$ is the power spectrum of the spike train.

${ }^{3}$ We do not impose a causality constraint on the filter $h$, since this requires solving the causal Wiener-Hopf equation (see Wiener 1949; Poor 1994, sect. V.D.2). Practically, $h$ has a finite support in the time domain and causality could also be implemented by introducing a delay in the reconstructions (Rieke 1991).
} 
where $S_{m}(\omega)$ is the power spectrum of the noise. From the definition of the noise,

$$
S_{n n}(\omega)=S_{s s}(\omega)-\frac{\left|S_{s x}(\omega)\right|^{2}}{S_{x x}(\omega)} \leq S_{s s}(\omega) .
$$

In this latter equation, $S_{s s}(\omega)$ is the power spectrum of the stimulus ensemble.

If we define the signal-to-noise ratio as

$$
\operatorname{SNR}(\omega)=\frac{S_{s s}(\omega)}{S_{m}(\omega)} \geq 1
$$

we may rewrite

$$
\epsilon^{2}=\frac{1}{2 \pi} \int_{-\omega_{c}}^{\omega_{c}} d \omega \frac{S_{S s}(\omega)}{S N R(\omega)}
$$

It follows from this latter equation that the mean square error in the reconstructions depends on the signal-to-noise ratio as well as on the bandwidth of the signal. The larger the signal-to-noise ratio, the smaller the mean square error. If, on the other hand, the signal-to-noise ratio is equal to 1 in some frequency band $\Delta$,

$$
\operatorname{SNR}(\omega)=1 \quad \text { for } \omega \in \Delta
$$

then the entire power of the signal in this frequency band contributes to the mean square error:

$$
\epsilon^{2}=\frac{1}{2 \pi} \int_{\left[-\omega_{c} ; \omega_{c}\right]-\Delta} d \omega \frac{S_{s s}(\omega)}{\operatorname{SNR}(\omega)}+\frac{1}{2 \pi} \int_{\Delta} d \omega S_{s s}(\omega) .
$$

In the extreme case where the spike train is completely unrelated to the signal,

$$
\operatorname{SNR}(\omega)=1 \quad \text { for all } \omega,|\omega| \leq \omega_{\mathrm{c}},
$$

it follows that the mean square error coincides with the variance $\sigma^{2}$ of the signal,

$$
\epsilon^{2}=\frac{1}{2 \pi} \int_{-\omega_{c}}^{\omega_{c}} d \omega S_{s s}(\omega)=\left\langle s(t)^{2}\right\rangle=\sigma^{2}
$$

From this we conclude that the relative mean error, defined as

$$
\epsilon_{\mathrm{r}}=\frac{\epsilon}{\sigma}
$$

is an appropriate measure of the quality of the reconstructions in the time domain, with $\epsilon_{\mathrm{r}}=1$ if the reconstructions are not better than chance and $\epsilon_{\mathrm{r}} \rightarrow 0$ in the limit of perfect reconstructions.

In earlier work on the subject, the reconstructions were quantified by using a different measure, the rate of information transmitted by $s_{\text {est }}(t)$ 
about $s(t)$ (Bialek et al. 1991; Rieke et al. 1993). The quantity computed in Bialek et al. (1991, equation 2) and Rieke et al. (1993, equation 3) is not an exact estimate of the mutual information rate $I\left(s_{\text {est }} ; s\right)^{4}$ between $s(t)$ and $s_{\text {est }}(t)$ but rather a lower bound, $I_{\mathrm{LB}}$,

$$
I\left(s_{\mathrm{est}} ; s\right) \geq I_{\mathrm{LB}}
$$

and the true rate of information transmission $I\left(s_{\text {est }} ; s\right)$ could take any value greater than or equal to $I_{\mathrm{LB}}$. It is possible to show that $I_{\mathrm{LB}}$ can be computed by using the simple formula,

$$
I_{\mathrm{LB}}=\frac{1}{4 \pi \log (2)} \int_{-\omega_{\mathrm{c}}}^{\omega_{\mathrm{c}}} d \omega \log [S N R(\omega)] \quad \text { (in bit/sec). }
$$

This avoids altogether the computation of the gain $g(\omega)$ introduced in Bialek et al. (1991) and Rieke et al. (1993) and thus simplifies and improves the numerical estimate of $I_{\mathrm{LB}} \cdot{ }^{5}$ Complete arguments regarding these two assertions can be found in Gabbiani (1995, section 3 and appendix I). As mentioned in the Introduction, in the case of the fly $\mathrm{H} 1$ neuron $I_{\mathrm{LB}}=$ $32 \mathrm{bit} / \mathrm{sec}$. The lower bound $I_{\mathrm{LB}}$ depends on the signal-to-noise ratio, but does not directly depend on the bandwidth of the signal. The frequency band

$$
\Delta=\{\omega \mid \operatorname{SNR}(\omega)>1\}
$$

contributes to information transmission whereas no information is transmitted if $S N R(\omega)=1$. It is hence clear from equations 2.3 and 2.6 that the mean square error and $I_{\mathrm{LB}}$ represent different measures of the reconstructions. This will be further illustrated in the numerical example of Section 4. A lower bound on the rate of information transmission per spike, $I_{S}$, can be obtained by dividing $I_{\mathrm{LB}}$ by the firing rate $\lambda$ of the cell:

$$
\left.I_{\mathrm{S}}=\frac{I_{\mathrm{LB}}}{\lambda} \quad \text { (in bit/spike }\right) .
$$

\section{A Simplified Model of Motion Encoding in H1 Neurons}

The lobula plate is a third-order neuropil in the visual system of the fly, which is believed to be a major motion computation center involved in visual course stabilization as well as visual fixation and discrimination of objects (Hausen and Egelhaaf 1989). In addition to small retinotopic elements, the lobula plate contains giant tangential cells that integrate motion signals over a large portion of the visual hemifields (Hausen

\footnotetext{
${ }^{4} I\left(s_{\text {est }} ; s\right)$ is itself a lower bound on the rate of information transmitted by the spike trains on the stimulus, see for example Rieke et al. (1993).

${ }^{5}$ The power spectrum of the stochastic noise process used in Bialek et al. (1991) and Rieke et al. (1993) can also be computed without introducing $8(\omega)$ (see Gabbiani 1995, appendix I).
} 
1984). H1 is a directionally selective cell belonging to the subclass of horizontal tangential neurons and is sensitive to horizontal back-to-front motion presented to the ipsilateral eye. ${ }^{6}$ It is believed that $\mathrm{H} 1$ is part of the neural circuitry underlying the optomotor response of flies (Eckert 1980), although its role is not clear since $\mathrm{H} 1$ is a heterolateral element connecting the two lobula plates and since it does not directly contact descending neurons that project to the motor centers of the thoracic ganglia (Strausfeld and Bassemir 1985). Nevertheless, the mean response of H1 has been shown to encode reliably the velocity contrast (or equivalently, contrast frequency contrast) of sinusoidal moving luminance patterns, at least at low modulation frequencies (Jian and Horridge 1991).

The stochastic activity of $\mathrm{H} 1$ in response to steady motion of sinusoidal gratings has been described by an integrate-and-fire model with random threshold under a wide range of velocities ${ }^{7}$ (Gestri et al. 1980). This is illustrated in Figure 1A: the stimulus $s(t)$ is first passed through a filter $F$ to yield the somatic current $\bar{q}_{s}(t)(\geq 0)$ (possibly after addition of a background current $q_{0}$, which describes the spontaneous activity of the cell). This current is then integrated to give the somatic voltage $y(t)$. When $y(t)$ reaches the threshold $k_{0}$, an action potential is fired and the voltage is reset to zero after an absolute refractory period $\delta$. The threshold is also reset to a new random value $k_{1}$ drawn from a given probability density distribution $p(k)$. In such models, the probability distribution $p(k)$ coincides up to constant factors with the interspike interval distribution of the cell in response to steady stimuli. It has been shown by Gestri et al. (1980) that $p(k)$ varies with the mean firing rate of the cell (see Fig. 1B) and these authors report an absolute refractory period in the range of 2-6 msec. The assumption of a random threshold distribution represents a convenient phenomenological description accounting for the trial-totrial variability of $\mathrm{H} 1$ spike trains in response to the same stimulus, but it is not meant to imply that variability is due to the spike generating mechanism. Indeed, it is likely that a substantial portion of this noise is presynaptic to H1 (Laughlin 1989; Bialek et al. 1991).

The integrate-and-fire model with random threshold has two further properties of interest:

1. The standard deviation $\pi$ of the interspike interval distribution (after subtraction of the absolute refractory period $\delta$, if $\delta \neq 0$ ) varies linearly with the mean interspike interval $\mu$ of the cell under steady stimuli (i.e., the coefficient of variation of the interspike interval distribution is independent of the mean firing rate). This fact is experimentally supported for H1 (Gestri et al. 1980; see also Fig. 1B, inset).

"Each thy has two $\mathrm{Hl}$ neurons, one in each lobula plate.

The directionally selective movement detector studied by Gestri et al. (1980) was not identified anatomically as being H1. However the response properties of H1 to visual stimuli are unique among horizontal tangential cells and are hence in principle sufficient to identify it. 
A

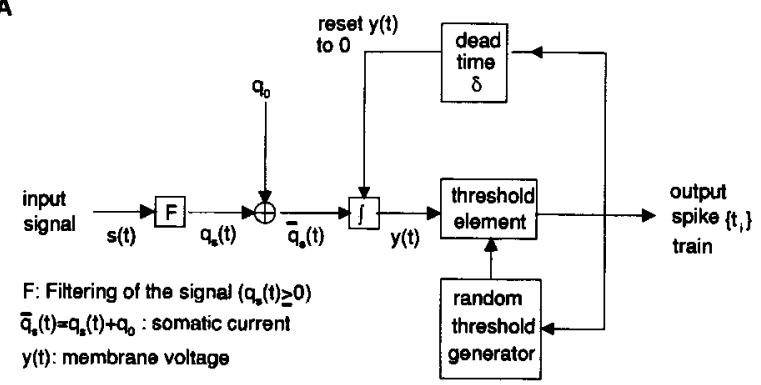

B
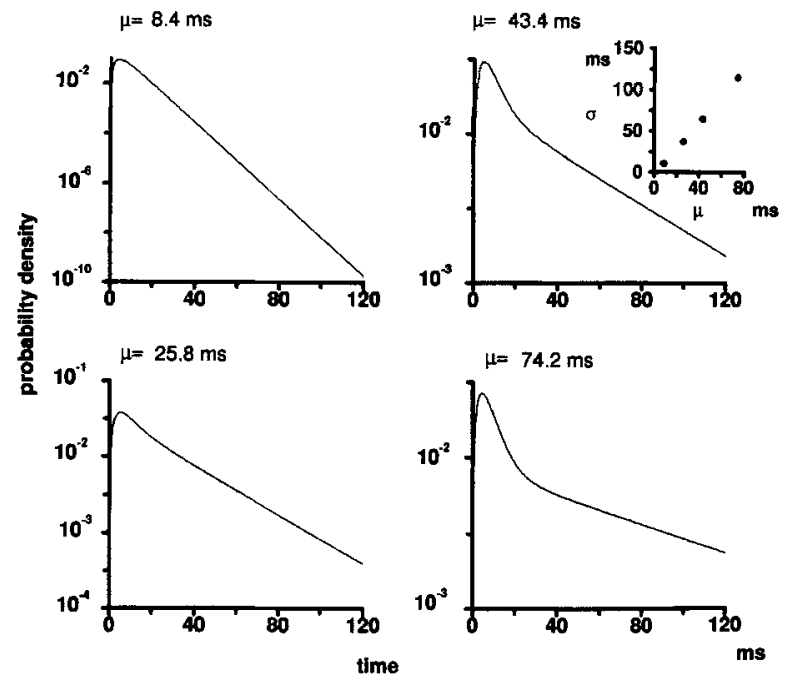

Figure 1: (A) schematic diagram illustrating the integrate-and-fire model with random threshold. The first box ( F ) represents the filtering of the input signal, which yields the positive current $q_{s}(t)$ injected into the cell. This current is then added to a current $q_{0}$ that represents the background activity of the cell and passed through an integrator $\left(\left[\int\right)\right.$ to yield the somatic voltage $y(t)$. When $y(t)$ reaches threshold a spike is generated, the voltage is reset to zero after an absolute refractory period $\delta$, and the threshold is reset to a new random value. (B) Experimentally measured change of the interspike interval probability density distribution of the $\mathrm{H} 1$ neuron with increase in the mean interspike interval (adapted from Gestri et al. 1980). The absolute refractory period $\delta=$ $4 \mathrm{msec}$ has been subtracted. The inset shows the standard deviation $(\sigma)$ of the interspike interval distribution as a function of the mean interspike interval $(\mu)$. 
2. The mean firing rate of an integrate-and-fire neuron with random threshold is proportional to the somatic current ${ }^{8} \bar{q}_{\mathrm{s}}(t)$ (Gestri $e t \mathrm{al}$. 1980) so that the model can, in principle, take into account the responses to velocity contrast observed in $\mathrm{H} 1$ (Jian and Horridge 1991).

We will make the following assumptions.

Assumption 1. The filter $F$ consists in a linear filtering by some filter $K$ of the velocity signal $s(t)$ followed by half-wave rectification,

$$
q_{\mathrm{s}}(t)=\int d t_{1} K\left(t_{1}\right) s\left(t-t_{1}\right) . \quad K\left(t_{1}\right) \geq 0
$$

and

$$
\begin{aligned}
& q_{s}^{+}(t)=\max \left\{q_{s}(t), 0\right\} . \\
& q_{s}^{-}(t)=\max \left\{-q_{s}(t), 0\right\} .
\end{aligned}
$$

with the positive currents $q_{\mathrm{s}}^{ \pm}(t)$ each driving one fictive H1 neuron; furthermore, we set $q_{0}=0$. This implements the directional selectivity of the H1 cells. Using an "opponency" principle, the output spike train of the two cells is

$$
x(t)=\sum_{i} \delta\left(t-t_{i}^{+}\right)-\sum_{i} \delta\left(t-t_{j}^{-}\right)
$$

with $\left\{t_{i}^{+}\right\}$encoding positive velocity and $\left\{t_{j}^{-}\right\}$encoding negative velocity.

To gain further insight into the meaning of the filter $K$, we study the response of the model to a step displacement of a pattern (that is, to a delta velocity pulse). If $s(t)=\delta\left(t-t_{0}\right)$ then

$$
\begin{aligned}
& q_{\mathrm{s}}^{+}(t)=K\left(t-t_{0}\right) \\
& q_{\mathrm{s}}^{-}(t)=0
\end{aligned}
$$

and, according to property 2 , the mean firing rate of one $\mathrm{H} 1$ model neuron will be proportional to $K\left(t-t_{0}\right)$. Hence, we may identify $K(t)$ with the mean firing rate of $\mathrm{H} 1$ in response to a step displacement of a pattern at time $t=0$. Such experiments have been performed on H1 (Srinivasan 1983) and it turns out that the response of the cell can be approximated (up to a pattern-dependent scale factor $\rho$ ) by an exponential low-pass

\footnotetext{
${ }^{8}$ This theoretical result is valid for any mean interspike interval $\mu>\delta$. In practice one expects it to hold true only if $\mu \gg \delta$.
} 
filter,

$$
K(t)= \begin{cases}0 & \text { if } t<0 \\ \rho e^{-t / \tau} & \text { if } t \geq 0\end{cases}
$$

with a time constant $\tau \cong 200$ msec. However, it has been subsequently shown that if $\mathrm{H1}$ is allowed to adapt to nonzero velocities (or more generally to temporal luminance modulation), the time constant $\tau$ of the filter $K$ shortens by more than one order of magnitude (Maddess and Laughlin 1985; de Ruyter van Steveninck et al. 1986; Borst and Egelhaaf 1987). To take this phenomena into account we assume in the following that $\tau=20 \mathrm{msec}$.

Since $\mathrm{H} 1$ integrates spatially the response of elementary motion detectors in response to a pattern moving in front of the animal, its mean firing rate will not in general be proportional to a low-pass filtered version of the horizontal component of the velocity vector, as in our model [see equation 3.1 and property 2 of the integrate-and-fire neurons with random threshold; Borst et al. 1993]. Hence, the model analyzed here is not a valid approximation for an arbitrary pattern presented to the fly. However, Reichardt and Schlögl (1988) showed that the output of horizontal elementary motion detectors (computed by assuming a small time delay between the response of two adjacent receptors and by considering only linear terms in the distance between the receptors) will be proportional to the horizontal component of the velocity for a sufficiently smooth pattern having independently distributed luminance along the horizontal and vertical axis (such as for the random pattern used by Bialek et al. 1991). Hence, in this approximation and for such patterns, the spatially integrated response of motion detectors will be proportional to the horizontal component of the velocity, with a pattern-dependent proportionality constant (see equation 3.4). As explained in equation 3.3, we also assume a low-pass filtering of the instantaneous velocity in our model. The range of velocities and velocity modulation for which this approximation is expected to hold will in general be pattern-dependent: it has been investigated by Egelhaaf and Reichardt (1987) for HS cells in the case of sinusoidal gratings. To the best or our knowledge, no equivalent experiments have been yet performed on $\mathrm{H} 1$.

Assumption 2. We neglect the absolute refractory period (and set $\delta=0$ in the following).

Assumption 3. We assume the threshold probability density distribution to be exponentially distributed,

$$
p(k) \propto e^{-k}
$$

Assumptions 2 and 3 represent a convenient first approximation of the response of $\mathrm{H} 1$ to velocity signals, since they allow us to compute analytically the optimal decoding filter $h$ for random velocity stimuli, as we report in the next section. 


\section{Results}

Under Assumptions 1 to 3 of the preceding section, the spike trains of our two model $\mathrm{H} 1$ neurons in response to a given velocity sample of the stimulus ensemble are equivalent to nonhomogeneous Poisson processes (Gestri 1971). This important simplification allows us to compute analytically the decoding filter $\widehat{h}(\omega)$ in the frequency domain. If $x(t)$ is given by equation 3.2 , we obtain,

$$
\begin{aligned}
\hat{h}(\omega) & =\frac{S_{s x}(-\omega)}{S_{x x}(\omega)} \\
& =\frac{\widehat{K}(-\omega) S_{s s}(\omega)}{\lambda_{K}+|\widehat{K}(\omega)|^{2} S_{s s}(\omega)},
\end{aligned}
$$

where

$$
S_{s x}(\omega)=\hat{K}(\omega) S_{s s}(\omega), \quad S_{x x}(\omega)=\lambda_{K}+|\hat{K}(\omega)|^{2} S_{s s}(\omega)
$$

and

$$
\lambda_{K}=\frac{1}{\pi}\left[\int_{-\omega_{c}}^{\omega_{i}} d \omega|\widehat{K}(\omega)|^{2} S_{s s}(\omega)\right]^{1 / 2}
$$

is the mean firing rate of both neurons (mean firing rate per neuron: $\lambda_{K} / 2$ ). The signal-to-noise ratio in the reconstructions (see Section 2) can be computed from these formulas,

$$
\operatorname{SNR}(\omega)=1+\frac{1}{\lambda_{K}}|\widehat{K}(\omega)|^{2} S_{S S}(\omega)
$$

and $I_{\mathrm{LB}}$ is then obtained using equation 2.6. For an exponential low-pass filter $K(t)$ (see last section) the Fourier transform $\hat{K}(\omega)$ is given by

$$
\widehat{K}(\omega)=\frac{\tau \rho}{1-i \omega \tau} .
$$

A result similar to equation 4.1 has been obtained for the first-order estimation of time-varying signals by saddle-point approximation in a model of a single neuron with an exponential dependence of the mean firing rate on the signal (Bialek 1989; Bialek and Zee 1990). drawn.

From the formula for $\widehat{h}(\omega)$ and $\operatorname{SNR}(\omega)$ several conclusions can be

1. The optimal linear reconstruction filter and the signal-to-noise ratio depend on the statistics of the stimulus in a nontrivial way. This implies that in our model, a change of the stimulus ensemble in the range of frequencies encoded by the cell will lead to a different filter $h$ and to a different signal-to-noise ratio.

2. The optimal linear filter $h$ and the signal-to-noise ratio depend on the firing rate of the neuron pair. This is most easily seen by scaling 
the filter $K$ by a positive constant $\eta$. If

$$
\widehat{K}(\omega) \rightarrow \widehat{K}^{(\eta)}(\omega)=\eta \widehat{K}(\omega), \quad \eta>0,
$$

then

$$
\lambda_{K} \rightarrow \lambda_{K}^{(\eta)}=\eta \lambda_{K}
$$

and

$$
\hat{h}(\omega) \rightarrow \hat{h}^{(\eta)}(\omega)=\frac{\widehat{K}(-\omega) S_{s s}(\omega)}{\lambda_{K}+\eta|\widehat{K}(\omega)|^{2} S_{s s}(\omega)} .
$$

We see from this last equation that the relative weighting of the two factors $\lambda_{K}$ and $|\widehat{K}(\omega)|^{2} S_{s s}(\omega)$ in the denominator depends on $\eta$ (or equivalently on the firing rate of the neurons, see equation 4.3). This implies that as the mean firing rate of the neurons changes, the shape of the decoding filter $h$ will change as well. The second term in equation 4.2 for the signal-to-noise ratio can be shown to depend linearly on the mean firing rate by a similar argument.

3. In the limit of low firing rates, the optimal linear decoding filter $h$ is given by the reverse-correlation of the spike train and the stimulus. This follows from equation 4.4 since as $\eta$ tends to zero,

$$
\widehat{h}^{(\eta)}(\omega) \rightarrow \frac{1}{\lambda_{K}} \widehat{K}(-\omega) S_{s s}(\omega)=\frac{1}{\lambda_{K}} S_{s x}(-\omega) \quad(\eta \rightarrow 0),
$$

so that Fourier transforming back,

$$
h^{(\eta)}(t) \rightarrow \frac{1}{\lambda_{K}} R_{s x}(-t) \quad(\eta \rightarrow 0) .
$$

This corresponds, up to a constant factor, to the reverse-correlation of the stimulus and the spike train.

As illustrated in the following example, these three effects are clearly seen for mean firing rates that are expected to be in the physiological range.

Numerical example. In Figure 2, a gaussian white noise stimulus with cut-off frequency $f_{c}=1000 \mathrm{~Hz}$ and a standard deviation $\sigma=132 \mathrm{deg} / \mathrm{sec}$ (Bialek et al. 1991) as well as sample spike trains generated by two H1 model neurons in response to the stimulus are shown. The mean firing rate of each neuron was $100 \mathrm{~Hz}$ and the time constant of the exponential low-pass filter $K$ was $\tau=20 \mathrm{msec}$ (see Section 3). Figure 3A-C shows the optimal decoding filters computed numerically and from equation 4.1 for the same stimulus and for model neurons firing at 5,50 , and $100 \mathrm{~Hz}$. As is clearly seen, the shape of the optimal decoding filter varies with the firing rate. For comparison, Figure 3D shows the decoding filter obtained for two model neurons firing at the same frequency as in Figure $3 \mathrm{C}$ ( $100 \mathrm{~Hz}$ per neuron), but in response to a white noise signal having a 
A

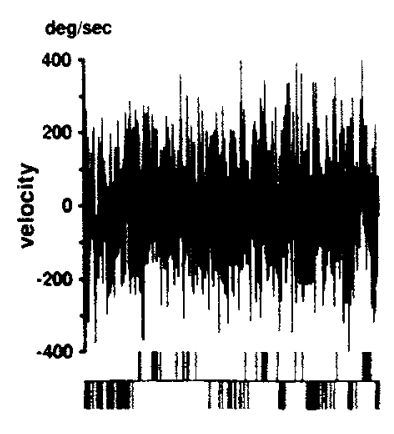

B

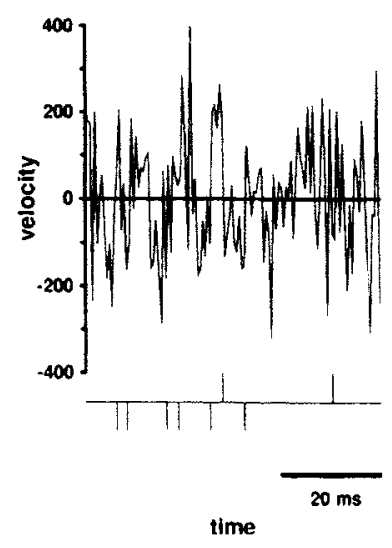

C

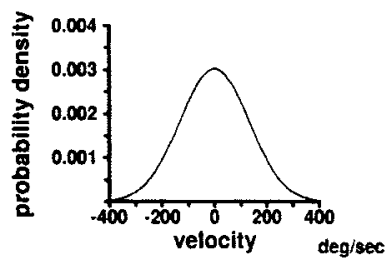

$\operatorname{deg}^{2} / \sec ^{2}$

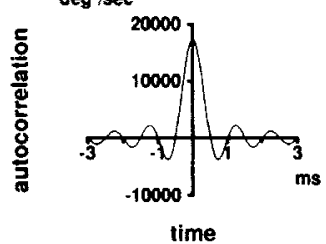

$\left(\operatorname{deg}^{2} / \sec ^{2}\right) \mathrm{Hz}$

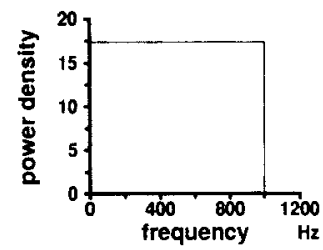

Figure 2: Sample spike trains generated by the model neurons in response to white noise. (A) The upper part of the graph shows the white noise velocity signal and the lower part the corresponding action potentials of two simulated, half-wave rectified neurons. Notice the occurrence of closely spaced action potentials (thicker lines) resulting trom the absence of a refractory period in our model. (B) A portion of the same signal and spike train as in $\mathrm{A}$ is shown at a magnified time scale. (C) Properties of the white noise stimulus $s(t)$. The upper graph shows the gaussian distribution of velocity (mean value: $0 \mathrm{deg} / \mathrm{sec}$, standard deviation: $132 \mathrm{deg} / \mathrm{sec}$ ), the middle graph the autocorrelation of the white noise, and the lower graph its one-sided power spectrum (cut-off frequency: $1000 \mathrm{~Hz}$. 

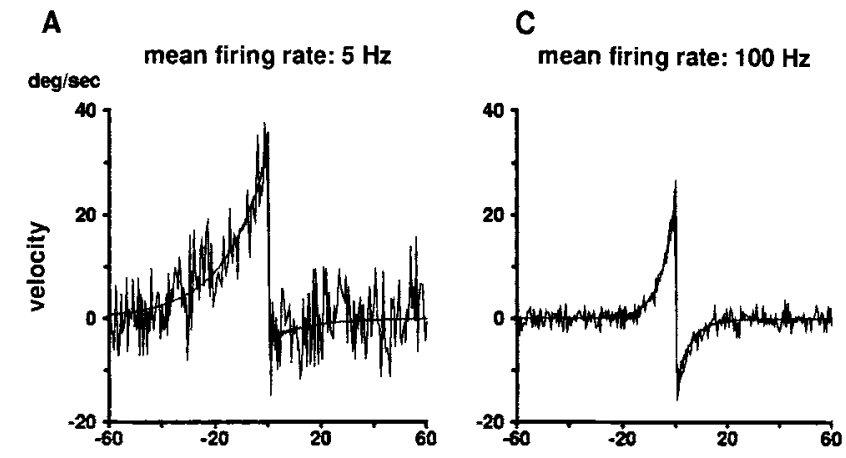

B
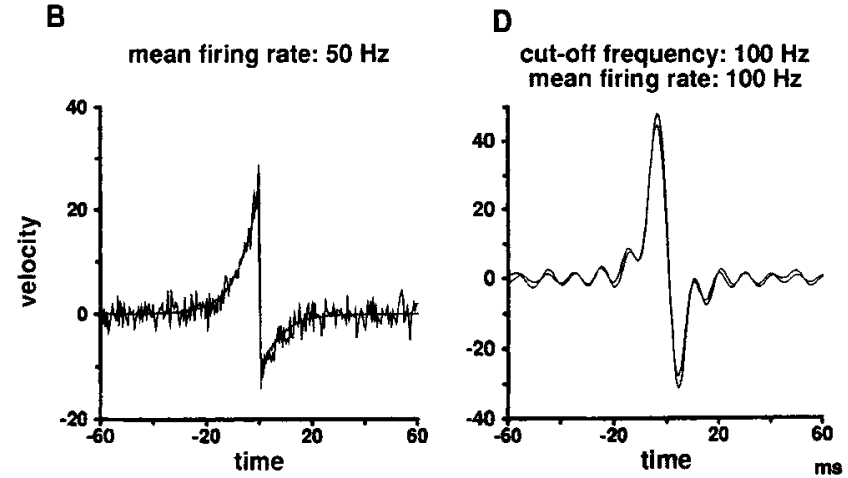

Figure 3: Comparison of optimal theoretical filters $h(t)$ with those obtained from numerical simulations at different firing rates. In simulations, the filters were calculated as explained in Section 2; 100 sweeps of $1 \mathrm{sec}$ each were used to compute $S_{s x}$ and $S_{x x}$. The smooth curves are the theoretical predictions and were obtained by numerically Fourier transforming equation 4.1 to the time domain. (A, B, and C) Filters obtained at mean firing rates $\left(\lambda_{K} / 2\right)$ of 5,50 , and $100 \mathrm{~Hz}$ per neuron (cut-off frequency of the white noise signal, $f_{\mathrm{c}}=1000 \mathrm{~Hz}$ ). The high-frequency noise in the numerical calculations decreases as the number of collected action potentials increases. Notice the progressive shortening of the integration window at negative times and the appearance of a negative velocity peak for $t>0$. (D) Filter obtained with a white noise signal having a cut-off frequency $f_{c}=100 \mathrm{~Hz}$ for two neurons firing each at a frequency of $100 \mathrm{~Hz}$. The high-frequency noise due to the poor encoding of high frequencies by the neuron models in $\mathrm{A}-\mathrm{C}$ has disappeared. 
cut-off frequency of $100 \mathrm{~Hz}$ (standard deviation of the signal in the time domain $\sigma=132 \mathrm{deg} / \mathrm{sec}$ ). As predicted by equation 4.1 , the change in statistics of the signal leads to a different filter than in Figure $3 \mathrm{C}$. Furthermore, the high-frequency noise contaminating the numerical filters of Figure $3 \mathrm{~A}-\mathrm{C}$ has disappeared (an explanation will be given below).

The lower bound $I_{\mathrm{LB}}$ (in bit/sec) on the rate at which the two neurons transmit information about the stimulus and the relative mean error $\left(\epsilon_{\mathrm{r}}=\sqrt{\epsilon^{2} / \sigma^{2}}\right.$, see Section 2$)$ can be estimated analytically and numerically (Gabbiani 1995, Sect. 6). The analytical calculation leads to

$$
\epsilon^{2}=\frac{\sigma^{2}}{\omega_{c}}\left[\omega_{c}-\frac{1}{\tau} \frac{\gamma}{\sqrt{1+\gamma}} \arctan \left(\frac{\tau \omega_{c}}{\sqrt{1+\gamma}}\right)\right]
$$

and

$$
\begin{aligned}
I_{\mathrm{LB}}= & \frac{1}{2 \pi \log (2)}\left[\omega_{\mathrm{c}} \log \left(1+\frac{\gamma}{1+\omega_{\mathrm{c}}^{2} \tau^{2}}\right)\right. \\
& \left.+\frac{2}{\tau} \sqrt{1+\gamma} \arctan \left(\frac{\tau \omega_{\mathrm{c}}}{\sqrt{1+\gamma}}\right)-\frac{2}{\tau} \arctan \tau \omega_{\mathrm{c}}\right]
\end{aligned}
$$

where

$$
\gamma=\frac{\pi^{2}}{2} \frac{\tau \lambda_{K}}{\arctan \tau \omega_{c}}
$$

Notice that in contrast to the mean square error $\epsilon^{2}$, the lower bound $I_{\mathrm{LB}}$ does not depend on the standard deviation $\sigma$ of the white noise signal in the time domain. Both $\epsilon_{\mathrm{r}}$ and $I_{\mathrm{LB}}$ vary with the mean firing rate $\left(\lambda_{K}\right)$ of the two neurons as well as with the cut-off frequency $\left(\omega_{c}\right)$ of the stimulus. From equations 4.5 and 4.6 it is easy to see that $\epsilon^{2}$ is a monotonically decreasing function of $\lambda_{K}$, whereas $I_{L B}$ is a monotonically increasing function of $\lambda_{K}$. In the limit of low firing rates, $\lambda_{K} \rightarrow 0$,

$$
\epsilon^{2} \rightarrow \sigma^{2} . \quad \epsilon_{\mathrm{r}} \rightarrow 1
$$

and

$$
I_{\mathrm{LB}} \rightarrow 0
$$

whereas for large firing rates, $\lambda_{K} \rightarrow \infty$,

$$
\epsilon^{2} \rightarrow 0, \quad \epsilon_{\mathrm{r}} \rightarrow 0
$$

and

$$
I_{\mathrm{LB}} \rightarrow \infty \text {. }
$$

The latter limit is, however, not an appropriate approximation for our system: $\epsilon_{\mathrm{r}}$ and $I_{\mathrm{LB}} / 2$ (the rate of information transmitted by a single neuron) are plotted as a function of the firing rate per neuron $\left(\lambda_{K} / 2\right)$ for a stimulus having a standard deviation $\sigma=132 \mathrm{deg} / \mathrm{sec}$ and a cut-off frequency 
A
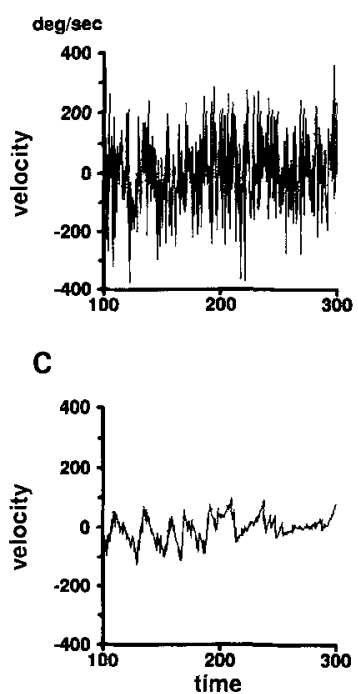

E

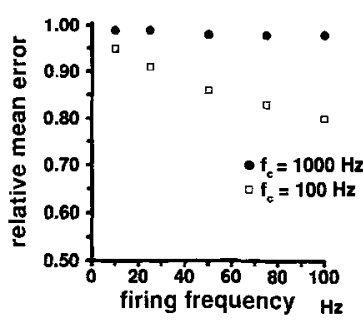

B

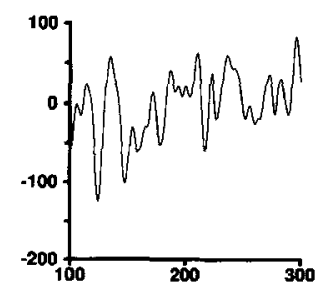

D

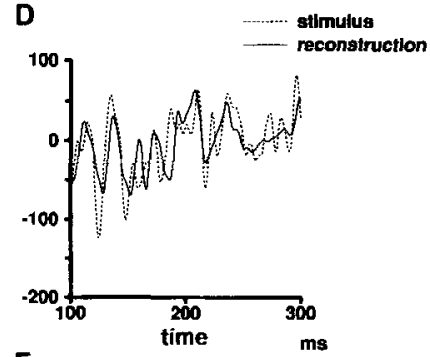

$F$

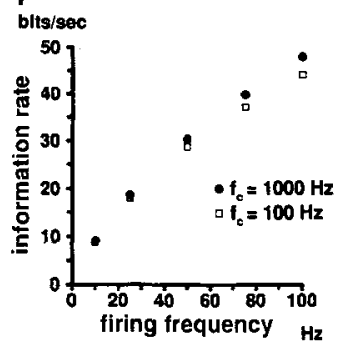

Figure 4: Comparison of signal and reconstructions before and after filtering with a $5 \mathrm{msec}$ half-width gaussian filter. (A) Original signal. (B) Same signal as in A, but smoothed using a $5 \mathrm{msec}$ half-width gaussian filter. (C) Reconstructed signal; relative mean error $\epsilon_{\mathrm{r}}=0.98$. (D) Superposition of the filtered signal and filtered reconstruction; relative mean error $\epsilon_{\mathrm{r}}=0.73$. (E, F) Relative mean error and rate of information transmission of each neuron $\left(l_{\mathrm{LB}} / 2\right.$, in bit/sec) as a function of the mean firing frequency per neuron $\left(\lambda_{K} / 2\right)$ for the particular stimulus ensemble shown in A (filled dots) and for an ensemble having the same standard deviation as in A, but a cut-off frequency of $100 \mathrm{~Hz}$ (squares). The choice of an appropriate stimulus bandwidth strongly decreases the mean square error without changing significantly $I_{\mathrm{LB}}$. 
$f_{c}=1000 \mathrm{~Hz}$ in Figure $4 \mathrm{E}$ and $\mathrm{F}$ (filled dots). We obtain $I_{\mathrm{LB}} / 2 \cong 50 \mathrm{bit} / \mathrm{sec}$ when each model neuron fires at a frequency of $100 \mathrm{~Hz}$. The relative mean error in the reconstructions is, however, very high, $\epsilon_{\mathrm{r}}=0.98$, indicating a poor reconstruction: only $1-0.98=2 \%$ of the stimulus is effectively encoded in the spike trains of the cells. This is further illustrated in Figure 4A and C. As observed by Bialek et al. (1991), if we smooth the stimulus and reconstructions with a $5 \mathrm{msec}$ half-width gaussian filter the quality of the reconstructions (as measured by $\epsilon_{\mathrm{r}}$ ) increases considerably to $\epsilon_{\mathrm{r}}=0.73$ (see Fig. $4 \mathrm{~B}$ and $\mathrm{D}$ ). In our model, this is a consequence of the low-pass filtering by $K$ of the stimulus s prior to the spike-generating mechanism. A time constant $\tau=20 \mathrm{msec}$ for the filter $K$ implies that frequencies in the signal above $100 \mathrm{~Hz}$ are strongly attenuated and not well encoded by the two model neurons. Hence, although the neurons transmit a significant amount of information in the frequency band from 0 to $100 \mathrm{~Hz}$, the quality of the reconstructions as measured by $\epsilon_{\mathrm{r}}$ cannot be significantly better than chance, since the neurons transmit almost no information in the frequency band between 100 and $1000 \mathrm{~Hz}$. This is in agreement with equations 2.3, 2.5, and 2.6 for $\epsilon_{r}$ and $I_{L B}$ presented in Section 2. By smoothing the stimulus and reconstructions with a $5 \mathrm{msec}$ half-width gaussian filter we strongly suppress the high frequencies in the stimulus ensemble, thereby revealing the improved accuracy of the reconstructions at low frequencies. As discussed in Section 3, a similar low-pass filtering is expected in the fly $\mathrm{H} 1$ cell and it has indeed been reported that in experimental reconstructions, the $\mathrm{H} 1$ neuron provides information about the stimulus mainly for frequencies below $25 \mathrm{~Hz}$ (Bialek t' al. 1991, Fig. 3). Similar or lower temporal cut-off frequencies in neurons responding to visual stimuli have also been observed in cat striate cortex and monkey area V1 (DeAngelis et al. 1993; Richmond et al. 1994). Finally, we plotted for the same firing rates in Figure $4 \mathrm{E}$ and $\mathrm{F}$ the relative mean error and lower bound on the information transmission rate for a white noise stimulus having the same standard deviation in the time domain as the previous one but with a cut-off frequency $f_{\mathrm{c}}=100 \mathrm{~Hz}$ (open squares). In both cases $\left(f_{c}=100\right.$ and $f_{c}=1000 \mathrm{~Hz}$ ), peak signal-to-noise ratios increase linearly with mean firing rate from a minimum of 1.5 at a firing rate $\lambda_{k} / 2=10 \mathrm{~Hz}$ up to a maximal value of 16 for $\lambda_{K} / 2=100 \mathrm{~Hz}$ (see equation 4.2). The rate of information transmission remains almost unchanged compared to the previous example (as expected from equation 2.6, since the signal still covers the frequency range in which the model neurons effectively transmit information) but the quality of the linear recontructions as measured by $\epsilon_{\mathrm{r}}$ improves significantly. Clearly, a reduction in the bandwith of the stimulus ensemble always leads to a reduction of the relative mean error $\epsilon_{\mathrm{r}}$ (see equation $4.5, \epsilon^{2} \rightarrow 0$ as $\omega_{c} \rightarrow 0$ ). However, as soon as the bandwith of the stimulus becomes smaller than the frequency range in which the cell transmits information, $I_{L B}$ decreases in parallel with $\epsilon_{\mathrm{r}}$ (in the limit $\omega_{\mathrm{c}}-0, I_{\mathrm{LB}}-0$ as well, see equation 4.6); this is not observed in the example of Figure $4 \mathrm{E}$ and $\mathrm{F}$. 
From equations 2.6 and 4.6 it is also possible to study the properties of the lower bound on the rate of information transmitted per spike, $I_{\mathrm{S}}=I_{\mathrm{LB}} / \lambda_{\mathrm{K}}$. In the example shown in Figure $4 \mathrm{~F}, I_{\mathrm{S}}$ ranges from $\sim 1 \mathrm{bit} / \mathrm{spike}$ (when each neuron fires at a rate $\lambda_{K} / 2=10 \mathrm{~Hz}$ ) down to $\sim 0.5 \mathrm{bit} / \mathrm{spike}$ (for $\lambda_{K} / 2=100 \mathrm{~Hz}$ ); these values are similar to those observed experimentally in $\mathrm{H} 1(\sim 0.75$ bit/spike; Rieke 1991). As can be seen in this example, $I_{S}$ is a monotonically decreasing function of the firing rate of the neurons. Furthermore, it can be shown that

$$
I_{S} \rightarrow 0 \quad\left(\lambda_{K} \rightarrow \infty\right)
$$

and

$$
I_{S} \rightarrow \frac{\pi}{4 \log (2)} \cong 1.13 \mathrm{bit} / \mathrm{spike} \quad\left(\lambda_{K} \rightarrow 0\right) .
$$

This latter limit is independent of the statistical properties of the stimulus and of the filter $K$ describing the linear processing of the signal prior to half-wave rectification and the spike mechanism. Hence, the model of a linear and half-wave rectifying neuron with exponentially distributed random threshold studied here cannot reproduce the higher values for $I_{S}(\sim 3 \mathrm{bit} /$ spike) observed experimentally in other preparations (Rieke et al. 1993).

\section{Discussion}

In this work, we analyzed and refined various aspects of the linear reconstruction method pioneered by Bialek and collaborators using analytical and numerical techniques. In brief, this method allows one to reconstruct the time-varying input signal from the spike train of a single neuron. We here argue that two different measures should be used to quantify the performance of this signal reconstruction method: the relative mean error, $\epsilon_{\mathrm{r}}$, and the lower bound on the rate of information transmitted by the estimated stimulus on the true stimulus, $I_{\mathrm{LB}}$.

To apply the methods discussed in this paper, the following assumptions need to be made: (1) both the stimulus as well as the spike train must be (weakly) stationary, (2) ensemble averages must be replaced by time averages in numerical calculations (that is, the spike trains of the neuron and the sample functions of the stimulus must be jointly ergodic), and (3) deterministic components in the stimulus or spike train samples need to be subtracted prior to the reconstruction (see footnote 1). In addition, the lower bound $I_{\mathrm{LB}}$ can be computed only by further assuming that the stimulus ensemble is gaussian and bandwidth limited. No such assumption needs to be made for the rest of the least-square signal reconstruction procedure. However, the "effective noise" introduced in Section 2 needs to be neither gaussian nor independent of the stimulus.

Using these assumptions, we derived a simplified model of motion encoding by $\mathrm{H} 1$ neurons based on the one developed by Gestri et al. 
(1980) to fit their experimental data. We also derived closed analytical formulas for the reconstruction filter in the frequency domain, the mean square error, the signal-to-noise ratio, and the lower bound on the rate of information transmission, and showed that for a plausible choice of parameters, our model reproduces qualitatively several aspects of the reconstruction experiments performed by Bialek $e$ al. (1991). Because of the simplifications that were made, our model can by no means reproduce all of their results. For example, a meaningful comparison of the noise in the reconstructions with the noise at the photoreceptor level (see Fig. 3 of Bialek et al. 1991) would require modeling the elementary motion detection circuitry presynaptic to $\mathrm{H} 1$ as well as including a more realistic distribution of the noise that was placed at the spiking threshold mechanism in Section 3. Nevertheless, the assumptions underlying our model have been clearly isolated, allowing it to be completely understood.

In Sections 2 and 4 , the quality of reconstructions was assessed by computing the relative mean error $\epsilon_{\mathrm{r}}$ (see equations 2.2, 2.5, and 4.5) in addition to the lower bound $I_{\mathrm{LB}}$ used in earlier works (Bialek et al. 1991; Rieke et al. 1993). Computing the mean relative error, as well as the rate of information transmission in different cases, allows us to study the significance of a measured rate of information transmission in terms of the reconstruction performance of the cell. Why use $\epsilon_{\mathrm{r}}$ to characterize the performance of the reconstruction method?

Two important properties of the relative mean error are (1) it can be computed under far less restrictive assumptions on the stimulus than $I_{\mathrm{LB}}$ and (2) it compares directly stimulus and reconstructions in the time domain. This is, of course, the primary reason why the least-square metric is commonly used in engineering and other applications, where a signal and a reconstructed or corrupted version of this signal need to be compared. In contrast, as shown by equation 2.6 and by the numerical example of Section $4, I_{L B}$ is able to reveal effective information transmission in a given frequency band but it does not compare directly the presented stimulus with the reconstructed one. It is for example possible for a model neuron to transmit $\sim 50 \mathrm{bit} / \mathrm{sec}$ of information about a time-varying stimulus, yet to reproduce less than $3 \%$ of that stimulus (see Fig. $4 \mathrm{~A}$ and $\mathrm{C}$ as well as $\mathrm{E}$ and $\mathrm{F}$ for the parameters $\sigma=132 \mathrm{deg} / \mathrm{sec}$, $f_{c}=1000 \mathrm{~Hz}$, and $\lambda_{K} / 2=100 \mathrm{~Hz}$ ). If, on the other hand, the cut-off frequency of the stimulus is chosen differently, the same model neuron firing at the same rate can now reproduce more than $20 \%$ of the stimulus for a similar value of $I_{\mathrm{LB}}$ (see Fig. $4 \mathrm{E}$ and $\mathrm{F}$ for the parameters $\sigma=132$ $\mathrm{deg} / \mathrm{sec}, f_{\mathrm{c}}=100 \mathrm{~Hz}$, and $\lambda_{K} / 2=100 \mathrm{~Hz}$ ). Hence, in these examples $I_{\mathrm{LB}}$ does not determine which portion of a time-varying stimulus is encoded in the spike train of a cell in the mean square sense. Furthermore, if the stimulus ensemble contains significant power in a frequency range that is not encoded by the cell, this part of the power spectrum contributes entirely to the mean square error (see Section 2, equation 2.4 and Section 4 , numerical example, $f_{c}=1000 \mathrm{~Hz}$ ). It is therefore necessary-to deter- 
mine which portion of a time-varying signal can be encoded by a cell in the mean square sense-to compute the experimental mean square error using equation 2.2 and to choose stimuli whose bandwidth is matched to the encoding possibilities of the recorded cell.

Finally, if more natural stimulus ensembles are used to drive our model neurons, the rates of information transmission $I_{\mathrm{LB}}$ obtained are lower than those obtained using white noise (Gabbiani 1995). In spite of this, the mean relative error in signal reconstructions decreases compared to white noise stimuli, showing that a better performance is achieved. This shows that both $I_{\mathrm{LB}}$ and $\epsilon_{\mathrm{r}}$ depend on the stimulus ensemble chosen to drive the cell and that while $I_{\mathrm{LB}}$ is likely to be overestimated by the choice of white noise stimuli, the performance of the cell $\left(\epsilon_{\mathrm{r}}\right)$ is likely to be underestimated.

The closed formula for the reconstruction filter of our model depends on the statistics of the stimulus ensemble as well as on the mean firing rate of the cell (see Section 4, properties 1 and 2). This theoretical result was shown to translate into changes of the reconstruction filter when the mean firing rate of the model was varied in the physiological range (see Fig. 3). We therefore expect that by using different stimulus ensembles in the range of frequencies encoded by the cell (for example, different bandwidths), one can demonstrate changes in the shape of experimental reconstruction filters. ${ }^{9}$ While in a natural environment the ensemble distribution of stimuli (such as velocity signals) encoded by a cell might not change significantly over time, the firing rate of a neuron is expected to change (with the mean contrast of the visual scene for example). This also implies in our model a change of the reconstruction filter. Hence, our results do not lend support to the idea that single synapses might serve as decoders of presynaptic spike trains, as suggested by Bialek et al. (1991), since the decoding algorithm might depend on additional parameters of the stimulus ensemble or on biophysical properties such as the firing rate of a cell. However, our results cannot be regarded as conclusive in this respect because effects that were not directly taken into account here (such as firing rate adaptation or saturation) might play an important role in determining the shape of $h$. In the case of the H1 neuron, for example, it will be of interest to relate the changes (or the invariance) of $h$ to the biophysical properties of $\mathrm{H} 1$ and its presynaptic elements.

As demonstrated by property 3 of Section 4 , the optimal reconstruction filter in the class of models considered here coincides with the reverse-correlation function in the limit of low firing rates. This rigorous result is of interest since it establishes a clear connection between reconstructions and the more traditional reverse-correlation method that has been extensively used to study the auditory and visual systems. It confirms the intuition that (in the limit of low firing rates) the optimal es-

\footnotetext{
${ }^{9}$ Up to now, only changes in the spatial characteristics of the stimulus ensemble have been tested (see Bialek et al. 1991).
} 
timate of the stimulus preceding a spike is given by reverse-correlation. Furthermore, it offers an explanation for the observation that reversecorrelation can be a successful method of reconstruction in certain cases (Gielen et al. 1988).

Finally, we wish to point out that the reconstructions performed on $\mathrm{H} 1$ in the house fly and on cells in other animals, as well as this theoretical work, leave totally open the important problem of determining whether the information on a time-varying stimulus that can be encoded in a neuronal spike train is actually used by the organism, i.e., the problem of correlating measures such as $I_{\mathrm{LB}}$ and $\epsilon_{\mathrm{r}}$ with the behavior of the animal.

\section{Acknowledgments}

We would like to thank J. Fröhlich and K. Hepp for very useful discussions on the subject treated here. The comments given by $W$. Bialek and $R$. de Ruyter van Steveninck on this manuscript are also gratefuly acknowledged. This work was supported by a grant of the Roche Research Foundation and in part by the Center for Neuromorphic Systems Engineering as a part of the National Science Foundation Engineering Research Center Program, and by the California Trade and Commerce Agency, Office of Strategic Technology.

\section{References}

Adrian, E. 1928. The Basis of Sensation; the Action of the Sense Organs. Christophers, London.

Bialek, W. 1989. Theoretical physics meets experimental neurobiology. In Lectures in Complex Systems, SFl Studie's in the Siences of Complexity, Vol. Il, E. Jen, ed., pp. 513-595. Addison-Wesley, New York.

Bialek, W. 1992. Optimal signal processing in the nervous system. In Princeton Lectures in Biophysics, pp. 321-401. World Scientific, Singapore.

Bialek, W., and Zee, A. 1990. Coding and computation with neural spike trains. J. Stat. Phys. 59(1), 103-115.

Bialek, W., de Ruyter van Steveninck, R., and Warland, D. 1991. Reading a neural code. Sciencie 252, 1854-1857.

Borst, A., and Egelhaaf, M. 1987. Temporal modulation of luminance adapts time constant of fly movement detectors. Biol. Cybern. 56, 209-215.

Borst, A., Egelhaaf, M., and Seung, H. 1993. Two-dimensional motion perception in flies. Neural Comp. 5, 856-868.

DeAngelis, G., Ohzawa, I., and Freeman, R. 1993. Spatiotemporal organization of simple-cell receptive fields in the cat's striate cortex. I. General characteristics and postnatal development. J. Neuroplysiol. 69, 1091-1117.

de Ruyter van Steveninck, R., Zaagman, W., and Masterbroeck, H. 1986. Adaptation of transient responses of a movement-sensitive neuron of the visual system of the bowfly Calliphora erythrocthaln. Biol. Cylom. 54, 223-236. 
Eckert, H. 1980. Functional properties of the H1-neuron in the third optic ganglion of the bowfly Phaenicia. J. Comp. Physiol. A 135, 29-39.

Eckhorn, R., Bauer, R., Jordan, W., Brosch, M., Kruse, W., Munk, M., and Reitboeck, H. 1988. Coherent oscillations: A mechanism of feature linking in the visual cortex? Biol. Cybern. 60, 121-130.

Egelhaaf, M., and Reichardt, W. 1987. Dynamic response properties of movement detectors: Theoretical analysis and electrophysiological investigation in the visual system of the fly. Biol. Cybern. 56, 69-87.

Gabbiani, F. 1995. Coding of time-varying signals in spike trains of linear and half-wave rectifying neurons (submitted).

Gestri, G. 1971. Pulse frequency modulation in neural systems, a random model. Biophys. J. 11, 98-109.

Gestri, G., Masterbroek, H. A. K., and Zaagman, W. H. 1980. Stochastic constancy, variability and adaptation of spike generation: Performance of a giant neuron in the visual system of the fly. Biol. Cybern. 38, 31-40.

Gielen, C., Hesselmans, G., and Johannesma, P. 1988. Sensory interpretation of neural activity patterns. Math. Biosci. 88, 15-35.

Gray, C., König, P., Engel, A., and Singer, W. 1989. Oscillatory responses in cat visual cortex exhibit inter-columnar synchronization which reflects global stimulus properties. Nature (London) 338, 1698-1702.

Hausen, K. 1984. The lobula-complex of the fly: Structure, function and significance in visual behavior. In Photoreception and Vision in Invertebrates, M. Ali, ed., pp. 523-559. Plenum Press, New York.

Hausen, K., and Egelhaaf, M. 1989. Neural mechanisms of visual course control in insects. In Facets of Vision, D. Stavenga and R. Hardie, eds., pp. 391-424. Springer-Verlag, Berlin.

Jian, S., and Horridge, G. 1991. The H1 neuron measures changes in velocity irrespective of contrast frequency, mean velocity or velocity modulation frequency. Phil. Trans. R. Soc. London B 331, 205-211.

Land, M., and Collett, T. 1974. Chasing behavior of houseflies (Fannia canicularis). A description and analysis. J. Comp. Physiol. 89, 331-357.

Laughlin, S. 1989. Coding efficiency and design in visual processing. In Facets of Vision, D. Stavenga and R. Hardie, eds., pp. 213-234. Springer-Verlag, Berlin.

Maddess, T., and Laughlin, S. B. 1985. Adaptation of the motion-sensitive neuron $\mathrm{H} 1$ is generated locally and governed by contrast frequency. Proc. $R$. Soc. London B 225, 251-275.

Middlebrooks, J., Clock, A., Xu, L., and Green, D. 1994. A panoramic code for sound location by cortical neurons. Science 264, 842-844.

Miller, M., and Sachs, M. 1983. Representation of stop consonants in the discharge patterns of auditory nerve fibers. J. Acoust. Soc. Am. 74(2), 502-517.

Moiseff, A., and Konishi, M. 1981. Neuronal and behavioral sensitivity to binaural time differences in the owl. J. Neurosci. 1, 40-48.

Oppenheim, A., and Schaffer, R. 1975. Digital Signal Processing. Prentice-Hall, Englewood Cliffs, NJ.

Optican, L., and Richmond, B. 1987. Temporal encoding of two-dimensional 
patterns by single units in primate inferior temporal cortex. J. Neurophysiol. $57,162-178$.

Poggio, G., and Viernstein, L. 1964. Time series analysis of impulse sequences of thalamic somatic sensory neurons. J. Neurophysiol. 27, 517-545.

Poor, H. 1994. An Introduction to Signal Detection and Estimation. Springer-Verlag, New York.

Rabiner, L., and Gold, B. 1975. Theory and Application of Digital Signal Processing. Prentice Hall, Englewood Cliffs, NJ.

Reichardt, W., and Schlögl, W. 1988. A two dimensional field theory for motion computation. First order approximation: Translatory motion of rigid patterns. Biol. Cybern. 60, 23-35.

Richmond, B., Heller, J., and Hertz, J. 1994. Neural response structure and dynamics of single neuronal information transmission in the visual system. In Proceedings of the International Symposium on Dynamics of Neural Processing, pp. 89-92. Washington, DC, June 6-8.

Rieke, F. 1991. Plysical principles underlying sensory processing and computation. Ph.D. thesis, University of California at Berkeley, Berkeley, CA.

Rieke, F., Warland, D., and Bialek, W. 1993. Coding efficiency and information rates in sensory neurons. Europhys. Lett. 22(2), 151-156.

Saleh, B. 1978. Photoelectron Statistics. Springer-Verlag, Berlin.

Simmons, J. 1979. Perception of echo phase information in bat sonar. Science 204, 1336-1338.

Softky, W., and Koch, C. 1993. The irregular firing of cortical cells is inconsistent with temporal integration of random EPSP's. J. Neturosci. 13, 334-350.

Srinivasan, M. 1983. The impulse response of a movement-detecting neuron and its interpretation. Vis. Res. 23(6), 659-663.

Strausfeld, N., and Bassemir, U. 1985. The organization of giant horizontalmotion-sensitive neurons and their synaptic relationships in the lateral deutocerebrum of Calliphora erythrocephala and Musca domestica. Cell Tissue Res. 242, 531-550.

Wiener, N. 1949. Extrapolation, Interpolation and Smoothing of Stationary Time Series. John Wiley \& Sons, New York.

Received August 2, 1994; accepted March 31, 1995. 